\title{
GENERIC REGULARITY OF HOMOLOGICALLY AREA MINIMIZING HYPERSURFACES IN EIGHT DIMENSIONAL MANIFOLDS
}

\author{
NATHAN SMALE
}

\section{INTRODUCTION}

It is a well known fact, due to Federer and Fleming [F-F], that if $N$ is a smooth, compact $(n+1)$-dimensional manifold, then for any nontrivial integer homology class $\alpha \in H_{k}(N, \mathbb{Z}), k \leq n$, there is a $k$-dimensional integer multiplicity rectifiable current $T$, of least area representing $\alpha$. One can think of $T$ as a (possibly singular) surface with multiplicity and orientation. Of course $T$ may have singularities for topological, or other reasons. There are however partial regularity results. Frederick Almgren [AF] has shown that in general, the singular set has Haussdorff dimension at most $k-2$, and Sheldon Chang [CS] further showed that if $k=2$ the singularities are isolated branch points. In codimension $1(k=n)$ it follows from the work of various people, including Federer, DeGiorgi, and Simons that $\operatorname{sing}(T)$ is empty for $n<7$ (see chapter 7 of [SL1], or chapter 3 of [FH1] for an exposition of this result), and Federer[FH2] proved that if $n \geq 7$ then the singular set has dimension at most $n-7$, and is disctrete in case $n=7$. It should be noted that in the codimension 1 case, there is no topological obstruction for the existence of a smooth minimizer in a given homology class; that is, given $\alpha \in H_{n}(N, \mathbb{Z})$ there is a smooth, oriented hypersurface $M$ (with multiplicity) homologous to $\alpha$. There are no known examples of singular homologically minimizing hypersurfaces, however one would certainly expect them to exist, since hypercurrents that minimize area with fixed boundary certainly can be singular (e.g. minimizing cones). The question that we are interested in here is whether for generic metrics on $N$, the minimizing current $T$ homologous to $\alpha$ is smooth. We state 
the following conjecture

Conjecture 0.1. Given a smooth, compact $(n+1)$ dimensional manifold $N$ with nonzero $n$ dimensional integral homology group, and given $\alpha \in H_{n}(N, \mathbb{Z})$, $\alpha \neq 0$, there exists an open, dense set $\mathcal{F}$, in the space of $C^{3}$ metrics, such that for $g \in \mathcal{F}$, there is a smooth area minimizing (relative to $g$ ) hypersurface $M$, homologous to $\alpha$.

The purpose of this paper is to prove this in the lowest dimension where singularities may occur, $n=7$. The reason the proof works in dimension 7 , is essentially that the structure of the singular set in this case is well understood, that is singularities are isolated and each singularity has a unique, regular tangent cone, to which $M$ converges to in fairly strong way [SL2]. We also use in a crucial way, a result of Hardt and Simon [H-S] which states that if $T$ is a locally area minimizing hypercurrent with $p \in \operatorname{spt}(T)$ and $\operatorname{sing}(T) \cap B_{\rho}(p)=$ $\{p\}$ where $B_{\rho}(p)$ is the geodesic ball in $N$ of radius $\rho$, and $T$ has a regular tangent cone at $p$, then any minimizing hypercurrent in $B_{\rho}(p)$ whose support is on one side of $\operatorname{spt}(T)$ (in particular, if $\partial T$ lies on one side), with boundary close to $\partial T \cap B_{\rho}(p)$, must be smooth. In fact, it follows from [H-S] that for generic boundaries in $\mathbb{R}^{8}$, there is a smooth area minimizing hypersurface with given boundary. One can of course form the analogous conjecture for minimizing hypercurrents with given boundary. In higher dimensions, very little is known. Rafe Mazzeo and the author [M-S], have some results in this direction, showing that certain kinds of higher dimensional singularities can be perturbed away, by a large family of boundary perturbations.

The idea of the proof is fairly simple. The openness condition is fairly straightforward, and follows from standard geometric measure theory, including the Allard regularity theorem, so most of the proof is devoted to showing that for a given metric $g_{0}$ on $N$ and minimizer $T$ homologous to $\alpha \in H_{7}(N, \mathbb{Z})$, there are nearby metrics such that the corresponding minimizer is smooth. The idea is to conformally perturb the metric so that the minimizer is pulled off to one side of $T$, and then use the results of [H-S].

The author would like to thank Rick Schoen, Leon Simon and Brian White for their interest and useful conversations on this problem. In fact, the au- 
thor had previously perturbed away the singularities with more complicated metrics, when Brian White pointed out to him that it could probably be done more simply with a conformal perturbation.

\section{ONE SIDED PERTURBATIONS OF THE METRIC}

Let $N$ be a smooth, compact, $(n+1)$-dimensional manifold with nontrivial $H_{n}(N, \mathbb{Z})$. For $k=3,4, \ldots$, let $\mathcal{M}^{k}$ denote the class of $C^{k}$ metrics on $N$, endowed with the $C^{k}$ topology, and let \|\|$_{k}$ be a norm defining the topology, (any other such norm of course being equivalent, as $N$ is compact). For $\alpha \in H_{n}(N, \mathbb{Z}), \alpha \neq 0$, we define the subclass $\mathcal{F}_{\alpha}^{k} \subset \mathcal{M}^{k}$, to be the set of metrics such that $g \in \mathcal{F}_{\alpha}^{k}$ if and only if there is a smooth area minimizing (relative to $g$ ) $n$-dimensional, integer multiplicity current $T$ homologous to $\alpha$. We also define the subclass $\mathcal{E}_{\alpha}^{k} \subset \mathcal{F}_{\alpha}^{k}$, to be the set of metrics in $\mathcal{M}^{k}$ that satisfy $g \in \mathcal{E}_{\alpha}^{k}$ if and only if there is a unique area minimizing, integer multiplicity current $T$ homologous to $\alpha$, and $T$ is smooth. Finally, set $\mathcal{F}^{k}=\bigcap_{\beta \in H_{n}} \mathcal{F}_{\beta}^{k}$; that is, the set of $C^{k}$ metrics such that every $n$-dimensional homology class admits a smooth, area minimizing representative. Of course, as mentioned above, any nontrivial homology class admits some area minimizing current as representative, by $[\mathrm{F}-\mathrm{F}]$. We will say that a subfamily $\mathcal{G} \subset \mathcal{M}^{k}$ is generic if it contains an open, dense set of $\mathcal{M}^{k}$. Our main result then, is

Theorem 1.1. For $n=7, \mathcal{F}_{\alpha}^{k}$ is generic in $\mathcal{M}^{k}$.

A subfamily $\mathcal{G} \subset \mathcal{M}^{k}$ will be called generic in the Baire sense if it is the countable intersection of generic subfamilies. A corollary of the theorem is then

Corollary 1.2. For $n=7, \mathcal{F}^{k}$ is generic in the Baire sense in $\mathcal{M}^{k}$.

This is clear, since $H_{n}(N, \mathbb{Z})$ is finitely generated. The main ingredient in the proof of the Theorem is

Main Lemma. For $n=7, \mathcal{E}_{\alpha}^{k}$ is dense in $\mathcal{M}^{k}$.

Assuming the main lemma for a moment, we can prove the Theorem as follows. If $g_{0} \in \mathcal{E}_{\alpha}^{k}$, with corresponding unique, smooth minimizer $T_{0}$ homologous to $\alpha$, we claim that there is a $\varepsilon=\varepsilon\left(g_{0}\right)>0$ such that if $\left\|g-g_{0}\right\|<\varepsilon$, then 
$g \in \mathcal{F}_{\alpha}^{k}$. If not, then there is a sequence $g_{j} \in \mathcal{M}^{k}$ with $g_{j} \rightarrow g_{0}$, with area minimizing currents $T_{j}$ (relative to $g_{k}$ ), and $\operatorname{sing}\left(T_{j}\right)$ nonempty. But, since $g_{j} \rightarrow g_{0}$, clearly $\operatorname{Mass}\left(T_{j}\right)$ is bounded independent of $j$, and so by compactness of integer multiplicity currents[F-F], and the fact that homology classes are weakly closed, there must be a subsequence, still denoted by $\left\{T_{j}\right\}$, and an integer multiplicity current $T$ homologous to $\alpha$, such that $T_{j} \rightarrow T$, in the sense of currents. By lower semi-continuity of mass, we have $\operatorname{mass}(T) \leq \operatorname{mass}\left(T_{0}\right)$, and so $T=T_{0}$, since $T_{0}$ is the unique homological minimizer. But since $T_{0}$ is smooth, it follows from Allards regularity theorem[AW], that if $x \in \operatorname{spt}(T)$ then there is a neighborhood $U$ of $x$ in $N$ such that for all sufficiently large $j, U \cap \operatorname{spt}\left(T_{j}\right)$, is smooth, contradicting the assumption that $\operatorname{sing}\left(T_{j}\right)$ was nonempty, and proving the claim. But then if we set $\mathcal{G}=\bigcup_{g \in \mathcal{E}_{\alpha}^{k}} \mathcal{B}_{\varepsilon(g)}(g)$, where $\mathcal{B}_{\sigma}(h)$ is the ball of radius $\sigma$ centered at $h$ in $\mathcal{M}^{k}$, it follows that $\mathcal{G} \subset \mathcal{F}_{\alpha}^{k}$ is a dense, open family in $\mathcal{M}^{k}$.

The proof of the Main Lemma is broken up into the following two density lemmas.

Lemma 1.3. Given $g_{0} \in \mathcal{M}^{k}$, and minimizing hypercurrent $T_{0}$ homologous to $\alpha$, and given $\varepsilon>0$, there is a metric $g \in \mathcal{M}^{k}$ such that $\left\|g-g_{0}\right\|_{k}<\varepsilon$, and $T_{0}$ is the unique minimizing current (relative to g) homologous to $\alpha$.

Lemma 1.4. Let $n=7$. Given $g_{0} \in \mathcal{M}^{k}$, with unique minimizing current $T_{0}$ homologous to $\alpha$, and given $\varepsilon>0$, there exists $g \in \mathcal{F}_{\alpha}^{k}$ with $\left\|g-g_{0}\right\|_{k}<\varepsilon$.

The Main Lemma is a consequence of Lemmas1.3 and 1.4 as follows. Given $g_{0} \in \mathcal{M}^{k}$ and $\varepsilon>0$, use Lemma 1.3 to find $g_{1} \in \mathcal{M}^{k}$, with $\left\|g_{1}-g_{0}\right\|_{k}<\varepsilon / 3$, such that the corresponding minimizer $T_{1}$ homologous to $\alpha$ is unique. Now apply Lemma 1.4 , to find $\gamma_{2} \in \mathcal{F}_{\alpha}^{k}$, with $\left\|g_{2}-g_{1}\right\|_{k}<\varepsilon / 3$, and let $T_{2}$ be a corresponding minimizer homologous to $\alpha$ which is smooth. Finally, apply Lemma 1.3 again, to find $g_{3} \in \mathcal{M}^{k}$ with $\left\|g_{3}-g_{2}\right\|_{k}<\varepsilon / 3$, such that $T_{2}$ is the unique minimizer homologous to $\alpha$. Such a $g_{3}$ is in $\mathcal{E}_{\alpha}^{k}$, and $\left\|g_{3}-g_{0}\right\|_{k}<\varepsilon$, showing that $\mathcal{E}_{\alpha}^{k}$ is dense in $\mathcal{M}^{k}$.

Remark 1.1. Before proving Lemmas 1.3 and 1.4, we make some general remarks on area minimizing currents. If $T$ is a $n$ dimensional integer multiplicity 
current in $(N, g)$, then $T=(M, d \mu, \xi)$, where $M$ is a $n$-dimensional rectifiable set, $d \mu=\theta d \mathcal{H}_{M}^{n}$ with $\theta(x) \in \mathbb{Z}$ the multiplicity, being $\mathcal{H}^{n}$ measurable, and $d \mathcal{H}_{M}^{n}$ denoting the $n$-dimensional Hausdorff measure on $M$. Also $\xi$ is a choice of orientation for the approximate tangent space of $M$ (which is $\mathcal{H}^{n}$ measurable). In our case (codimension 1 ) it follows from the regularity theory mentioned in the introduction that $M=\bigcup_{i=1}^{K} M_{i}$, where $M_{i}$ are the components of $M$, and $M_{i} \backslash S_{i}$ is a smooth hypersurface where $S_{i} \subset M_{i}$ has Haussdorff dimension $\leq 7$, and is isolated if $n=7$. Furthermore, $\theta(x) \equiv \theta_{i}$ on $M_{i}$ and with appropriate choice of $\xi$ we can assume that $\theta_{i}>0$. Also, clearly the multiplicity 1 current $\left(M_{i}, d \mathcal{H}_{M_{i}}^{n}, \xi\right)$ is homologically area minimizing (or else $T$ wouldn't be). The mass of $T$ is just the area of $M$ (with multiplicity)

$$
\mathbf{M}(T)=\int_{M} \theta(x) d \mathcal{H}^{n}(x)=\sum_{i=1}^{K} \theta_{i} \int_{M_{i}} d \mathcal{H}^{n}
$$

See either chapters 6 and 7 of [SL1] or [FH1] for a good reference on the theory of currents.

Much of the proof does not require that $n=7$, and so we will fix $n=7$ only where needed.

Proof of Lemma 1.3. Let $g_{0} \in \mathcal{M}^{k}$ and let $T_{0}$ be an area minimizing current (relative to $g_{0}$ ) homologous to $\alpha \in H_{n}(N, \mathbb{Z}), \alpha \neq 0$. Thus $T_{0}$ is as in the remark, $T_{0}=(M, d \mu, \xi)$ with $M=\bigcup_{j=1}^{N} M_{j}$. For each $M_{j}, j=1, \ldots, N$, we will perform a conformal perturbation of $g_{0}$ in a neighborhood of a regular point. Basically, we will 'pinch' $N$ about $M_{j}$. Let $p_{j} \in \operatorname{reg}\left(M_{j}\right)$, and let $\rho>0$ be small enough so that $B_{\rho}\left(p_{j}\right) \cap M_{j} \subset \operatorname{reg}(M)$ and so that we have well defined Fermi coordinates $(r, x)$ in $B_{\rho}\left(p_{j}\right)$, where $x=\left(x_{1}, \ldots, x_{n}\right)$ are normal coordinates on $M_{j}$ centered at $p_{j}$, and $r$ is the signed distance from $M_{j}$ (that is $\rho$ is chosen small enough so that $M_{j}$ divides $B_{\rho}\left(p_{j}\right)$ into two pieces and we have chosen one side to have $r>0$ ). Let $\eta$ be a non negative bump function on $M$ with the properties

$$
\eta(x)=1 \text { for } x \in B_{\rho / 2}\left(p_{j}\right) \cap M_{j}, \text { and } \eta(x)=0 \text { for } x \in B_{\rho}\left(p_{j}\right) \backslash B_{3 \rho / 4}\left(p_{j}\right) \text {. }
$$

We will denote $\operatorname{supp}(\eta)$ by $\Omega$. Now let $\phi_{0}: \mathbb{R} \rightarrow \mathbb{R}$ be a smooth function with 
the properties

$$
\begin{aligned}
& \operatorname{supp}(\phi) \subset[-3 / 4,3 / 4] \\
& \phi(r) \geq 0, \quad \text { for all } r \in[-1,1] \\
& \phi(0)=1, \quad \text { and } \phi(r)<1 \quad \text { if } r \neq 1 .
\end{aligned}
$$

Finally, for some fixed $t>k$ we let $\phi_{\varepsilon}(r)=\varepsilon^{t} \phi_{0}(r / \varepsilon)$, for all $\varepsilon>0$. Evidently, there is a $\varepsilon_{0}>0$ such that if $\varepsilon \leq \varepsilon_{0}$, the function $(r, x) \rightarrow \phi_{\varepsilon}(r) \eta(x)$, where $(r, x)$ are Fermi coordinates as above, is supported in $\bigcup_{j=1}^{K} B_{3 \rho / 4}\left(p_{j}\right)$, and so we can define the following function on all of $M$

$$
u_{\varepsilon}=1-\phi_{\varepsilon} \eta \text {. }
$$

We now defined our perturbed metrics to be $g_{\varepsilon}=u_{\varepsilon}^{2 / n} g_{0}$, for $\varepsilon \leq \varepsilon_{0}$, and we have the following

Proposition 1.5. There is a $\varepsilon_{1}>0$ such that for $0<\varepsilon \leq \varepsilon_{1}, T_{0}$ is the unique area minimizer, relative to $g_{\varepsilon}$, and homologous to $\alpha$.

Proof. This is intuitively obvious, since we have decreased the area of $\operatorname{spt}\left(T_{0}\right)$ strictly more than any competing hypersurface. The proof uses cutting and pasting arguments, and the definition of $\phi_{\varepsilon}$, and is very similar to the proof of Lemma 1.4, so we will omit the precise details. Basically, if $S$ is any hypersurface in $N$, then

$$
A_{\varepsilon}(S)=\int_{S}\left(1-\phi_{\varepsilon} \eta\right) d A_{0}
$$

where $A_{\varepsilon}$ is the $n$-dimensional Hausdorff measure relative to $g_{\varepsilon}$, and $\phi_{\varepsilon}$ is strictly maximized on $M$ (where $r=0$ ).

Proof of Lemma 1.4. Here we use a somewhat different conformal perturbation, one that will force the minimizer off to one side. Let $g_{0} \in \mathcal{M}^{k}$, such that there is a unique minimizing current $T_{0}$ homologous to $\alpha \in H_{n}(N, \mathbb{Z}$ ) (in a later part of the argument we will require that $n=7$ ). As in the proof of Lemma 1.3 , let $T_{0}=(M, d \mu, \xi)$ where $M=\operatorname{spt}\left(T_{0}\right)=\cup_{i=1}^{K} M_{i}$ and $M_{i}$ are the connected components of $M$, and let $B_{\rho}\left(p_{i}\right)$ be pairewise disjoint, geodesic balls centered at regular points $p_{i}$ of $M_{i}$, with Fermi coordinates $(r, x)$ so that $\xi$ points to the side of $M_{i}$ with $r>0$. Note that $M_{i}$ with multiplicity one is the unique homological area minimizer in its homology class. We also let 
$\eta=\eta(x)$ be as in the proof of Lemma 1 and $\Omega=\operatorname{supp}(\eta)$. For $\rho_{0}>0$ to be fixed later, we let $\phi_{0}$ be a smooth function $\phi_{0}: \mathbb{R} \rightarrow \mathbb{R}$ with the following properties

$$
\begin{aligned}
\phi_{0}(-s) & =-\phi_{0}(s) \quad \text { for } s \in \mathbb{R} \\
\phi_{0}(s) & \geq 0 \quad \text { for } s \geq 0 \\
\phi_{0}(s) & =s \quad \text { for } 0 \leq s \leq \rho_{0} / 4 \\
\phi_{0}(s) & =\rho_{0} / 2 \quad \text { for } \rho_{0} / 2 \leq s \leq 3 \rho_{0} / 4 \\
\phi_{0}(s) & =0 \quad \text { for } s \geq \rho_{0} .
\end{aligned}
$$

A crucial property of $\phi_{0}$, is that it is linear in a neighborhood of zero. We now fix $\rho_{0}$ so small that $(r, x) \rightarrow \phi_{0}(r) \eta(x)$ is supported in $\bigcup_{i=1}^{K} B_{\rho}\left(p_{i}\right)$. Also, fix some $t \in(0,1)$, and define for $\varepsilon \in(0,1)$ the function $\phi_{\varepsilon}(r)=\varepsilon^{t} \phi_{0}(r)$. We finally define the function $u_{\varepsilon}: M \rightarrow \mathbb{R}$ by

$$
\begin{aligned}
& u_{\varepsilon}(y)=1-\phi_{\varepsilon}(r) \eta(x) \text { for } y \in \bigcup_{i=1}^{K} B_{\rho}\left(p_{i}\right), \\
& u_{\varepsilon}(y)=1 \text { for } y \notin \bigcup_{i=1}^{K} B_{\rho}\left(p_{i}\right),
\end{aligned}
$$

and we let $g_{\varepsilon} \in \mathcal{M}^{k}$ be the metric $g_{\varepsilon}=u_{\varepsilon}^{2 / n} g_{0}$. We will finish the proof of the lemma by showing that for all sufficiently small $\varepsilon$, there is a smooth minimizing current $T$ (relative to $g_{\varepsilon}$ ), homologous to $T_{0}$. Suppose on the contrary, that there was a sequence $\varepsilon_{j} \rightarrow 0$, and corresponding homologically area minimizing currents $T_{\varepsilon_{j}}$ relative to $g_{\varepsilon_{j}}$, and homologous to $T_{0}$, with $\operatorname{sing}\left(T_{\varepsilon_{j}}\right) \neq \emptyset$. Clearly, $\mathbf{M}\left(T_{\varepsilon_{j}}\right) \leq c$ for some constant $c$ independent of $\varepsilon$ since $g_{\varepsilon} \rightarrow g_{0}$ in $C^{k}$, thus it follows from compactness of minimizing currents in a homology class (and the above convergence of metrics) that there is a subsequence, still denoted by $T_{\varepsilon_{j}}$ such that $T_{\varepsilon_{j}} \rightarrow T$, for some homologically minimizing current $T$ relative to $g_{0}$, homologous to $T_{0}$. Since $T_{0}$ is the unique such current, we must have $T=T_{0}$. Now $T_{\varepsilon_{j}}=\left(P^{j}, d \mu^{j}, \xi^{j}\right)$ as in the remark above, $P^{j}$ denoting the support of $T_{\varepsilon_{j}}, d \mu^{j}$ the $n$-dimensional Hausdorff measure (with multiplicity) on $P^{j}$ and $\xi^{j}$ an orientation on the approximate tangent spaces of $P^{j}$, and of course $P^{j}$ satisfying the regularity properties mentioned in the remark. We will use the result of Hardt and Simon to show that each $P^{j}$ must be a regular 
hypersurface for $j$ sufficiently large, getting a contradiction. As in the above remark, each $P_{j}$ decomposes into a union of sheets of multiplicity 1 , each being homologically area minimizing. For each $j$, let $Q_{j}$ be such a sheet, with $\operatorname{sing}\left(Q_{j}\right) \neq \emptyset$, and $y_{j} \in \operatorname{sing}\left(Q_{j}\right)$. By the area minimizing property, we must have $Q_{j} \rightarrow M$ in Hausdorff distance, and thus $Q_{j} \rightarrow Q$ in Hausdorff distance, where $Q$ is one of the sheets of $T_{0}$, (that is, one of the $M_{i}$ ). By the Allard regularity theorem[AW], this convergence is smooth away from $\operatorname{sing}(Q)$, and thus (after taking a subsequence if necessary) $y_{j} \rightarrow y_{0}$ for some $y_{0} \in \operatorname{sing}(Q)$. Let $\mathcal{N}$ be a distance neighborhood of $Q$ in $N$, such that $M \cap \mathcal{N}=Q$, and so that $Q$ divides $\mathcal{N}$ into two disjoint, open pieces, $\mathcal{N}_{+}$, and $\mathcal{N}_{-}$, where $\mathcal{N}_{+}$and $\mathcal{N}_{-}$correspond to $r>0$ and $r<0$ respectively. To finish the proof of the Lemma, we note that it suffices to prove the following proposition (we have simplified notation, setting $\phi_{j}=\phi_{\varepsilon_{j}}$ )

Proposition 1.6. For sufficiently large $j, Q_{j}$ has the following properties:

$$
\begin{array}{ll}
\text { A. } & Q_{j} \cap \mathcal{N}_{-}=\emptyset \\
\text { B. } & Q_{j} \cap \mathcal{N}_{+} \neq \emptyset \\
\text { C. } & Q_{j} \cap \mathcal{N}_{+} \backslash \operatorname{supp}\left(\phi_{j} \eta\right) \neq \emptyset .
\end{array}
$$

The proposition, together with Leon Simon's maximum principle[SL3] imply that $Q_{j} \backslash \operatorname{supp}\left(\phi_{j} \eta\right) \subset \mathcal{N}_{+} \backslash \operatorname{supp}\left(\phi_{j} \eta\right)$. Then, if $j$ is large enough, $Q_{j}$ can be made as close as we want to $Q$ in Hausdorff distance, and so it follows from Theorem 5.6 of [H-S] that $Q_{j}$ must be smooth (since $g_{\varepsilon}=g_{0}$ on $N \backslash \operatorname{supp}\left(\phi_{j} \eta\right)$ ), thus finishing the proof of the Lemma. Of course property $\mathrm{C}$ implies $\mathrm{B}$ but the proof of $\mathrm{C}$ requires the result of $\mathrm{B}$.

Proof of the Proposition. Let $\Sigma \subset \mathcal{N}$ be a union of open balls containing $\operatorname{sing}(Q)$, of sufficiently small radii such that $\Sigma \cap \operatorname{supp}\left(\phi_{0} \eta\right)=\emptyset$. Then, since $Q_{j} \rightarrow Q$ smoothly, away from $\Sigma$, there exist $C^{3}$ functions $f_{j}: Q \backslash \Sigma \rightarrow \mathbb{R}$, such that in Fermi coordinates $Q_{j} \backslash \Sigma$ is given by $\left\{(r, x): r=f_{j}(x), x \in Q \backslash \Sigma\right\}$, and $\left|f_{j}\right|_{C^{3}} \rightarrow 0$ as $j \rightarrow \infty$. To prove property A, note that

$$
A_{\varepsilon_{j}}\left(Q_{j}\right)=A_{0}\left(Q_{j}\right)-\int_{Q_{j}} \phi_{\varepsilon} \eta d A_{0}
$$


where $A_{\varepsilon_{j}}$, and $A_{0}$ denote the $n$-dimensional Hausdorff measure relative to $g_{\varepsilon_{j}}$ and $g_{0}$ respectively. Also of course $\phi_{\varepsilon_{j}} \eta \leq 0$ on $\mathcal{N}_{-}$. Now if A were false, then there would exist a nontrivial connected component $\hat{Q}_{j}$ of $Q_{j} \cap \mathcal{N}_{-}$, and we can assume that $\hat{Q}_{j}$ has nonempty boundary $\partial \hat{Q}_{j} \subset Q$ (otherwise $Q_{j} \subset \mathcal{N}_{-}$ and so $Q_{j}$ would be smooth by the above remarks and [H-S]). Let $\hat{Q}$ be the subset of $Q$ homologous to $\hat{Q}_{j}$ with $\partial \hat{Q}=\partial \hat{Q}_{j}$, and let $\tilde{Q}_{j}=\left(Q_{j} \backslash \hat{Q}_{j}\right) \cup \hat{Q}$. Then,

$$
\begin{aligned}
A_{\varepsilon_{j}}\left(\tilde{Q}_{j}\right) & =A_{0}\left(\tilde{Q}_{j}\right)-\int_{\tilde{Q}_{j}} \phi_{\varepsilon} \eta d A_{0} \\
& \leq A_{0}\left(Q_{j}\right)-\int_{\tilde{Q}_{j}} \phi_{\varepsilon} \eta d A_{0} \\
& =A_{0}\left(Q_{j}\right)-\int_{Q_{j} \backslash \hat{Q}_{j}} \phi_{\varepsilon} \eta-\int_{\hat{Q}} \phi_{\varepsilon} \eta d A_{0} \\
& \leq A_{0}\left(Q_{j}\right)-\int_{Q_{j} \backslash \hat{Q}_{j}} \phi_{\varepsilon} \eta-\int_{\hat{Q}_{j}} \phi_{\varepsilon} \eta d A_{0} \\
& =A_{\varepsilon_{j}}\left(Q_{j}\right)
\end{aligned}
$$

where the first inequality holds because $A_{0}(\hat{Q}) \leq A_{0}\left(\hat{Q}_{j}\right)$, and the second because $\phi_{\varepsilon} \eta=0$ on $\hat{Q}(r=0)$, and $\phi_{\varepsilon} \eta \leq 0$ on $\hat{Q}_{j}$ where $r<0$. But then the multiplicity one current associated to $\tilde{Q}_{j}$ is also $g_{\varepsilon}$-homologically area minimizing. However, it is easy to see that $Q$ must intersect $Q_{j}$ transversally along $\partial \hat{Q}_{j}$, and thus $\tilde{Q}_{j}$ has a codimension 1 singularity, contradicting the fact that it is area minimizing, thus proving property $\mathrm{A}$.

Due to the result of $\mathrm{A}$, it suffices in proving $\mathrm{B}$ to construct a $n$-dimensional rectifiable varifold $Q_{j}^{\prime}$, whose associated current is homologous to $Q$, such that $Q_{j}^{\prime} \cap \mathcal{N}_{+} \neq \emptyset$ and $A_{\varepsilon_{j}}\left(Q_{j}^{\prime}\right)<A_{\varepsilon_{j}}(Q)$. We will construct such a $Q_{j}^{\prime}$ by cutting and pasting from $Q$ inside $\operatorname{supp}\left(\phi_{0} \eta\right)$. Let $s \in\left(0, \rho_{0} / 8\right)$, and let $h_{s}: Q \rightarrow \mathbb{R}$ be a function with the following properties

$$
\begin{aligned}
& 0 \leq h_{s}(x) \leq s \\
& h_{s}(x)=s, \text { for } x \in \cup_{p_{i} \in Q} B_{\rho_{0} / 8}\left(p_{i}\right) \\
& h_{s}(x)=0 \text { for } x \in Q \backslash \cup_{p_{i} \in Q} B_{\rho_{0} / 4}\left(p_{i}\right), \\
& \left|\nabla h_{s}\right|(x) \leq c \text { for some } c \text { independent of } \mathrm{s} \text {, and } x \in Q .
\end{aligned}
$$

Now define the varifold $Q_{s}$ in Fermi coordinates about $Q$ by $Q_{s}=\left\{\left(h_{s}(x), x\right)\right.$ : 
$x \in Q\}$. Clearly $Q_{s} \subset \overline{\mathcal{N}}_{+}$for $s$ small, $Q_{s} \cap \mathcal{N}_{+} \neq \emptyset$ and the multiplicity one current associated to $Q_{s}$ is homomlogous to $Q$. Note that by the linearity property of $\phi_{0}$ near 0 , and the definition of $Q_{s}$, we have

$$
c_{2} s \geq \int_{Q_{s}} \phi_{0} \eta d A_{0} \geq c_{1} s,
$$

for some constants $c_{1}$, and $c_{2}$, and furthermore, by the first variation formula we must have

$$
A_{0}\left(Q_{s}\right)=A_{0}(Q)+O\left(s^{2}\right) .
$$

Then it follows from the definition of $\phi_{\varepsilon}$ that

$$
\begin{aligned}
A_{\varepsilon_{j}}\left(Q_{s}\right) & =A_{0}\left(Q_{s}\right)-\varepsilon_{j}^{t} \int_{Q_{s}} \phi_{0} \eta d A_{0} \\
& \leq A_{0}(Q)+O\left(s^{2}\right)-c_{1} \varepsilon_{j}^{t} s
\end{aligned}
$$

and therefore if we set $s=\varepsilon_{j}$, we get

$$
\begin{aligned}
A_{\varepsilon_{j}}\left(Q_{\varepsilon_{j}}\right) & \leq A_{0}(Q)+c_{2}\left(\varepsilon_{j}^{2}-\varepsilon_{j}^{t+1}\right), \\
& <A_{0}(Q)
\end{aligned}
$$

for $\varepsilon_{j}$ sufficiently small, since $t<1$. As observed, this finishes the proof of statement B.

To prove statement C, we need to show that $Q_{j}$ does not coincide with $Q$ outside of $\operatorname{supp}\left(\phi_{0} \eta\right)$. Suppose, on the contrary, that $Q_{j} \backslash \Omega=Q \backslash \Omega$. Then, it follows from properties $\mathrm{A}$ and $\mathrm{B}$ above, that the function $f_{j}$ defined at the beginning of the proof, whose graph is $Q_{j}$, is compactly supported within $\Omega$, $f_{j} \geq 0$ on $\Omega$ and $f_{j}$ is not identically zero on $\Omega$. That is, $Q_{j}$ lies on the $\mathcal{N}_{+}$side of $Q$, and comes in tangentially to $Q$ inside $\operatorname{supp}\left(\phi_{0} \eta\right)$. Now, for $\sigma \in(0,1)$, define $\Omega_{\sigma}=\{x \in \Omega: \operatorname{dist}(x, \partial \Omega) \geq \sigma\}$ and let $\Sigma_{j, \sigma}=\left\{\left(x, f_{j}(x)\right): x \in \partial \Omega_{\sigma}\right\}$. From the above remark, there exists $\sigma$ (for each $j$ ), so that $\Sigma_{j, \sigma}$ lies above $\partial \Omega_{\sigma}$, and does not coincide with $\partial \Omega_{\sigma}$. Now, clearly $Q_{j, \sigma}=\left\{\left(x, f_{j}(x): x \in \Omega \backslash \Omega_{\sigma}\right\}\right.$ is the $g_{\varepsilon_{j}}$-area minimizing current with boundary $\partial \Omega \cup \Sigma_{j, \sigma}$. Let $R_{j, \sigma}$ be the $g_{0}$-area minimizing current with boundary $\partial \Omega \cup \Sigma_{j, \sigma}$ which is homologous to $Q_{j, \sigma}$. It follows from Allards regularity theorem, that for $j$ sufficiently large, 
$R_{j, \sigma}$ is the graph over $\Omega \backslash \Omega_{\sigma}$ of some $C^{3}$ function $h_{j}$. Of course, we have

$$
\begin{aligned}
& h_{j} \geq 0 \text { on } \Omega \backslash \Omega_{\sigma} \\
& h_{j} \not \equiv 0 \text { on } \Omega \backslash \Omega_{\sigma} \\
& h_{j} \equiv 0 \text { on } \partial \Omega, \text { and } h_{j}=f_{j} \text { on } \partial \Omega_{\sigma} .
\end{aligned}
$$

We now claim that $h_{j} \leq f_{j}$ on $\Omega \backslash \Omega_{\sigma}$. Note that this would finish the proof of $\mathrm{C}$, since this would imply that $R_{j, \sigma}$ is tangent to $\Omega \backslash \Omega_{\sigma}$ along $\partial \Omega$ and lies on one side of $\Omega \backslash \Omega_{\sigma}$, and therefore by the maximum principle (since they are both minimizing relative to $g_{0}$ ) would have to coincide with $\Omega \backslash \Omega_{\sigma}$ contradicting the fact that $f_{j} \not \equiv 0$. However, the claim follows easily from the definition of $\phi_{j}$. Assume that the claim was false, and let $\Lambda$ be a component of $\left\{x \in \Omega \backslash \Omega_{\sigma}\right.$ : $\left.h_{j}(x)>f_{j}(x)\right\}$, and let $\hat{R}_{j, \sigma}$ and $\hat{Q}_{j, \sigma}$ denote the graphs of $h_{j}$ and $f_{j}$ over $\Lambda$ respectively. But then, since

$$
1-\phi_{j}\left(f_{j}(x)\right) \eta(x)<1-\phi_{j}\left(h_{j}(x)\right) \eta(x) \text { for } x \in \Lambda,
$$

and $\hat{R}_{j, \sigma}$ has smaller $g_{0}$-area than $\hat{Q}_{j, \sigma}$ it easily follows that

$$
\begin{aligned}
A_{\varepsilon_{j}}\left(\hat{R}_{j, \sigma}\right) & =\int_{\hat{R}_{j, \sigma}}\left(1-\phi_{j}\left(h_{j}(x)\right) \eta(x) d A_{0}(x)\right. \\
& <\int_{\hat{Q}_{j, \sigma}}\left(1-\phi_{j}\left(f_{j}(x)\right) \eta(x) d A_{0}(x)=A_{\varepsilon_{j}}\left(\hat{Q}_{j, \sigma}\right),\right.
\end{aligned}
$$

contradicting the fact that $Q_{j}$ is homologically area minimizing relative to $g_{\varepsilon}$, proving the claim, and thus completing the proof of part $\mathrm{C}$ of the proposition. As remarked earlier, this completes the proof of the Theorem.

It should be remarked that the only place in the proof, that we used $n=$ 7 , was where we invoked Theorem 5.6 of [H-S] to conclude that $Q_{j}$ was smooth. In particular, if the result of [H-S] was extended to $n \geq 7$, then the proof given here would imply the conjecture for $n \geq 7$. Unfortunately, not much is known in this direction for higher dimensions, although some partial results are given in [M-S].

\section{REFERENCES}

[AW] Allard, W. K., On the first variation of a varifold, Ann. Math. 95 (1972), 417-491. 
[AF] Almgren, F. J., Q valued functions minimizing Dirichlet's integral and the regularity of area minimizing rectifiable currents up to codimension two, Preprint.

[CS] Chang, S. X.-D., Two dimensional area minimizing currents are classical minimal surfaces, J. Amer. Math. Soc. 1 (1988), 699-778.

[FH1] Federer, H., Geometric Measure Theory, Springer-Verlag, Berlin-HeidelbergNew York 1969.

[FH2] Federer, H., The singular sets of area minimizing rectifiable currents with codimension one and of area minimizing flat chains modulo two with arbitrary codimension, Bull. Amer. Math. Soc., 76 (1960), 767-771.

[F-F] Federer, H., and Flemming, W. Normal and integral currents, Ann. Math. 72 (1960), 458-520.

[H-S] Hardt, R., and Simon, L., Area minimizing hypersurfaces with isolated singularities, J. Reine Angew. Math. 362 (1985), 102-129 .

[M-S] Mazzeo, R., and Smale, N., Perturbing away higher dimensional singularities from area minimizing hypersurfaces, Preprint 1991.

[SL1] Simon, L., Lectures on Geometric Measure Theory, Proceedings for the Centre for Mathematical Analysis, Australian National University, Canberra 1983.

[SL2] Simon, L., Asymptotics for a class of nonlinear evolution equations, with applications to geometric problems, Ann. Math. 118 (1983), 525-571.

[SL3] Simon, L., A strict maximum principle for area minimizing hypersurfaces, J. Differential Geom. 26 (1987), 327-335.

UNIVERSITY OF UTAH, U. S. A.

RECEIVED OCTOBER 1, 1993. 